\title{
Preparation, Physicochemical Characterization, and Cell Viability Evaluation of Long-Circulating and pH-Sensitive Liposomes Containing Ursolic Acid
}

\author{
Sávia Caldeira de Araújo Lopes, ${ }^{1}$ Marcus Vinícius Melo Novais, ${ }^{1}$ Cláudia Salviano Teixeira, ${ }^{1}$ \\ Kinulpe Honorato-Sampaio, ${ }^{2}$ Márcio Tadeu Pereira, ${ }^{3}$ Lucas Antônio Miranda Ferreira, ${ }^{1}$ \\ Fernão Castro Braga, ${ }^{1}$ and Mônica Cristina Oliveira ${ }^{1}$ \\ ${ }^{1}$ Departamento de Produtos Farmacêuticos, Faculdade de Farmácia, Universidade Federal de Minas Gerais, \\ Avenida Antônio Carlos, 6627, 31270-901 Belo Horizonte, MG, Brazil \\ ${ }^{2}$ Centro de Microscopia, Universidade Federal de Minas Gerais, Avenida Antônio Carlos, 6627, 31270-901 Belo Horizonte, \\ MG, Brazil \\ ${ }^{3}$ Centro de Desenvolvimento de Tecnologia Nuclear (CDTN)/Comissão Nacional de Energia Nuclear (CNEN), \\ Avenida Antônio Carlos, 6627, 31270-901 Belo Horizonte, MG, Brazil
}

Correspondence should be addressed to Mônica Cristina Oliveira; monica@farmacia.ufmg.br

Received 4 January 2013; Accepted 8 July 2013

Academic Editor: Andrei Surguchov

Copyright (C) 2013 Sávia Caldeira de Araújo Lopes et al. This is an open access article distributed under the Creative Commons Attribution License, which permits unrestricted use, distribution, and reproduction in any medium, provided the original work is properly cited.

\begin{abstract}
Cancer is one of the leading causes of death worldwide. Although several drugs are used clinically, some tumors either do not respond or are resistant to the existing pharmacotherapy, thus justifying the search for new drugs. Ursolic acid (UA) is a triterpene found in different plant species that has been shown to possess significant antitumor activity. However, UA presents a low solubility in aqueous medium, which presents a barrier to its biological applications. In this context, the use of liposomes presents a promising strategy to deliver UA and allow for its intravenous administration. In this work, long-circulating and $\mathrm{pH}$ sensitive liposomes containing UA (SpHL-UA) were developed, and their chemical and physicochemical properties were evaluated. SpHL-UA presented adequate properties, including a mean diameter of $191.1 \pm 6.4 \mathrm{~nm}$, a zeta potential of $1.2 \pm 1.4 \mathrm{mV}$, and a UA entrapment of $0.77 \pm 0.01 \mathrm{mg} / \mathrm{mL}$. Moreover, this formulation showed a good stability after having been stored for 2 months at $4^{\circ} \mathrm{C}$. The viability studies on breast (MDA-MB-231) and prostate (LNCaP) cancer cell lines demonstrated that SpHL-UA treatment significantly inhibited cancer cell proliferation. Therefore, the results of the present work suggest the applicability of SpHL-UA as a new and promising anticancer formulation.
\end{abstract}

\section{Introduction}

Ursolic acid (UA) is a triterpenoid compound that exists abundantly in the plant kingdom. UA has been reported to have an interesting bioactivity, including anti-inflammatory $[1,2]$, antihyperlipidemic $[3,4]$, and hepatoprotective [3] properties. Recent studies have shown that UA has revealed antitumor effects and cytotoxic activity towards various types of cancer cell lines [5-12].

However, although UA presents the advantage of low toxicity, the clinical application of UA is limited due to several problems, such as its limited water solubility, which leads to a low bioavailability and poor pharmacokinetics in vivo and subsequently restricts its effectiveness [13]. Another limitation is its nonspecific distribution throughout the body when administered intravenously. Thus, it is desirable to explore novel formulations of UA that overcome these inconveniences [12]. In this context, the use of nanosystems as carriers, such as liposomes, consists of a promising strategy to deliver this substance and allow for its intravenous administration. Moreover, considering the antitumor action of this triterpene, the use of nanocarriers as vehicles can enable the 
targeting of this compound to the tumor region, providing greater therapeutic efficacy.

Liposomes are well-recognized drug delivery systems which can act as biocompatible, biodegradable, and nonimmunogenic drug carriers [14]. A major drawback of conventional liposomes is the rapid uptake of these particles in vivo by cells of the mononuclear phagocyte system (MPS) $[15,16]$. Several different strategies have been developed to overcome these difficulties, such as coating the surface of the liposomes with inert molecules, like polyethylene glycols (PEG), which form a spatial barrier. The presence of PEG on the surface of the liposomal carrier has proven to extend blood-circulation time while reducing MPS uptake (stealth liposomes). This technology has resulted in a large number of nanocarriers encapsulating active molecules, with high target efficiency and activity [17-19].

In spite of the strategies mentioned previously, conventional and long-circulating liposomes may present a slow release of the active substance or may be unable to fuse with the endosome after internalization. As such, polymorphic liposomes have been developed to overcome these problems, mainly due to the fact that these liposomes become reactive when submitted to membrane changes triggered by $\mathrm{pH}$, variations in temperature, or surface charge alterations. A $\mathrm{pH}$-sensitive liposome is generally stable at physiological $\mathrm{pH}$ but can undergo destabilization and acquire fusogenic properties under acid conditions, thus leading to the release of its aqueous contents [20-22]. The development of this kind of liposome was proposed after the observation that some pathological tissues, including tumors or areas of inflammation and infection, as compared to normal tissues, reveal an acid environment [23]. Furthermore, the endosome formed during the cellular internalization of liposomes also presents an acidic $\mathrm{pH}$ which favors their fusion and release of entrapped drugs [21].

Therefore, in the present study, long-circulating and $\mathrm{pH}$ sensitive liposomes consisting of dioleoylphosphatidylethanolamine (DOPE), cholesteryl hemisuccinate (CHEMS), and distearoylphosphatidylethanolamine-polyethyleneglyc$\mathrm{ol}_{2000}$ (DSPE-PEG 2000 ) containing UA (SpHL-UA) were developed and their chemical and physicochemical properties were evaluated. In addition, the effect of SpHL-UA towards cancer cell lines viability, such as MDA-MB-231 and LNCaP, was also investigated.

\section{Materials and Methods}

2.1. Materials. DOPE and DSPE-PEG ${ }_{2000}$ were supplied by Lipoid GmbH (Ludwigshafen, Germany). UA, CHEMS, phosphate saline buffer, sodium hydroxide, Triton X-100, sodium dodecyl sulfate (SDS), MTT reagent (3-(4, 5-dimethylthiazolyl-2)-2,5-diphenyltetrazolium bromide), fetal bovine serum (FBS), penicillin, and streptomycin were obtained from Sigma Chemical Company (St. Louis, MO, USA). Roswell Park Memorial Institute Medium 1640 (RPMI) and Dulbecco's Modified Eagle's Medium (DMEM) were purchased from Gibco (Grand Island, NE, USA). Methanol was obtained from Fisher Scientific (NJ, USA). All other chemicals used in this study were of analytical grade.

The cancer cell line MDA-MB-231 (human breast adenocarcinoma) was kindly supplied by Professor Dr. Alfredo Miranda de Goes (Laboratory of Biochemistry and Immunology, Institute of Biological Sciences, Universidade Federal de Minas Gerais, Belo Horizonte, Brazil). The LNCaP (human prostate carcinoma) cancer cell was purchased from American Type Culture Collection (ATCC) (Manassas, VA, USA).

2.2. Preparation of SpHL-UA. For SpHL-UA preparation, the present study employed the lipid hydration method described by Bangham et al. [24]. Briefly, $11.4 \mathrm{~mL}$ of DOPE $50 \mathrm{mM}, 7.6 \mathrm{~mL}$ of CHEMS $50 \mathrm{mM}$, and $5 \mathrm{~mL}$ of DSPE$\mathrm{PEG}_{2000} 10 \mathrm{mM}$, dissolved in chloroform, were transferred to a round bottom flask amounting a total lipid concentration of $20 \mathrm{mM}$ (molar ratio of $5.7: 3.8: 0.5$, resp.). Similarly, $22.8 \mathrm{~mL}$ of DOPE $50 \mathrm{mM}, 15.2 \mathrm{~mL}$ of CHEMS $50 \mathrm{mM}$, and $10 \mathrm{~mL}$ of DSPE-PEG $_{2000} 10 \mathrm{mM}$, dissolved in chloroform, in a total lipid concentration of $40 \mathrm{mM}$ (molar ratio of $5.7: 3.8: 0.5$, resp.) were also transferred to a round bottom flask. UA equivalent at $0.1 \%(\mathrm{w} / \mathrm{v})$ or $0.05 \%(\mathrm{w} / \mathrm{v})$ was added to the lipid solution. A lipid film was obtained by evaporating the chloroform under reduced pressure. Next, the lipid film was hydrated with an amount of $3.8 \mathrm{~mL}$ of $\mathrm{NaOH} 0.1 \mathrm{M}$, for $20 \mathrm{mM}$ liposomes, and $7.6 \mathrm{~mL}$ of $\mathrm{NaOH} 0.1 \mathrm{M}$ for $40 \mathrm{mM}$ liposomes to promote the complete ionization of CHEMS. Finally, phosphate buffersaline (PBS), pH 7.4, was added in amount of $46.2 \mathrm{~mL}$ for $20 \mathrm{mM}$ liposomes and $42.4 \mathrm{~mL}$ for $40 \mathrm{mM}$ liposomes. The obtained mixture was subjected to vigorous shaking in a vortex, producing multilamellar liposomes. The resulting multilamellar vesicles were calibrated using a single-stage high pressure homogenizer, model APV 2000 (APV, Albertslund, Denmark) in recirculation mode. The pressure of the homogenizer was adjusted to 500 bar. The minimum volume of processed samples was $110 \mathrm{~mL}$, and all homogenizations were carried out at room temperature. Each cycle was equal to the passage of the total volume of the sample through the homogenization chamber in a total of twelve cycles. Nonentrapped UA was separated by ultrafiltration, using a polyethersulfone membrane (Millipore Pellicon XL device; Biomax, cut off $500 \mathrm{kDa}$; MA, USA) connected to a tangential flow filtration system (Labscale; Millipore, MA, USA). The liposome suspension $(100 \mathrm{~mL})$ was transferred to an initial container and pumped to the filtration membrane. Nonentrapped UA (permeated) was recovered in a flask, while purified SpHL-UA was returned to the initial container. This process was maintained until a concentrated SpHL-UA suspension reached the final volume of $25 \mathrm{~mL}$. After the cycle of ultrafiltration was completed, the permeated was taken and the nonentrapped UA concentration was measured by high performance liquid chromatography (HPLC). In addition, the purified SpHL-UA was diluted with PBS buffer $\mathrm{pH} 7.4$ to the final volume of $50 \mathrm{~mL}$. Next, the evaluation of drug entrapment was performed by HPLC (see Section 2.3.1).

In order to prepare sterile SpHL-UA, samples were irradiated at $10 \mathrm{kGy}$, for about 30 minutes (dose rate of $20.57 \mathrm{kGy} / \mathrm{h}$ ), using a multipurpose panoramic irradiator 
equipped with a Cobalt-60 source (IR-214 model, MDS Nordion, Canada) at the Center for the Development of Nuclear Technology-National Commission on Nuclear Energy (CDTN-CNEN, Brazil).

\subsection{SpHL-UA Characterization}

2.3.1. Drug Entrapment Determination. The evaluation of drug entrapment was performed after having purified the SpHL-UA, which was solubilized by adding methanol. The total amount of UA in the liposomes was also measured after SpHL-UA had been dissolved in methanol, before purification procedures by ultrafiltration. After preparing the samples, as mentioned previously, UA quantification was performed by HPLC. The chromatographic apparatus consisted of a model 515 pump, a model 717 Plus autoinjector, and a model 2487 variable wavelength UV detector (Waters Instruments, USA) controlled by Empower software. Separations were performed using a $25 \mathrm{~cm} \times 4 \mathrm{~mm}, 5 \mu \mathrm{m}$ LiChrosorb, RP-18 column (Merck SA, Germany). The eluent system consisted of a 1:1 methanol/water mixture, and the flow rate was $1.5 \mathrm{~mL} \cdot \mathrm{min}^{-1}$. Samples $(20 \mu \mathrm{L})$ were injected, and the absorbance of the eluate was monitored at $210 \mathrm{~nm}$.

The specificity of the HPLC method was carried out by comparing the peak retention time of UA and the peaks obtained after having injected liposomes without UA (blank liposomes). The linear response was evaluated in the concentration range of $5-60 \mu \mathrm{g} / \mathrm{mL}$ of UA. The precision of the method was assessed considering repeatability and intermediate precision at three concentration levels of UA (5, 20 , and $60 \mu \mathrm{g} / \mathrm{mL}$ ) on three different days.

2.3.2. Measurements of Size and Zeta Potential. The mean diameter of SpHL-UA was determined by photon correlation spectroscopy (PCS) at $25^{\circ} \mathrm{C}$ and at an angle of $90^{\circ}$. The zeta potential was evaluated by determining the electrophoretic mobility at an angle of $90^{\circ}$. The measurements were performed using the Zetasizer 3000 HAS (Malvern Instruments Ltd, Worcestershire, UK). The samples were diluted with PBS buffer solution.

2.3.3. Transmission Electron Microscopy (TEM). Morphological examination of SpHL-UA was performed by means of TEM using a negative staining method. The liposomes were placed on a formvar-coated copper grid and stained with a $2 \%(\mathrm{w} / \mathrm{v})$ phosphotungstic acid solution containing $0.5 \%$ $(\mathrm{w} / \mathrm{v})$ bovine serum albumin and $0.5 \%(\mathrm{w} / \mathrm{v})$ saccharose. The stained samples were characterized using a Tecnai G2 12 Spirit Biotwin FEI at $80 \mathrm{kV}$ (Centro de Microscopia, Universidade Federal de Minas Gerais, Belo Horizonte, Brazil).

2.3.4. Stability Study. The determination of the storage stability of SpHL-UA was performed at 15, 30, and 60 days after preparation. These formulations were maintained at $4^{\circ} \mathrm{C}$. The parameters evaluated included mean diameter, zeta potential, and drug entrapment. The mean values of these parameters were compared with those obtained at time zero.

\subsection{In Vitro Studies}

2.4.1. Cell Cultures. The effect of SpHL-UA on cell viability was evaluated on cancer cell lines MDA-MB-231 and LNCaP. Cells were cultured in RPMI 1640 for LNCaP cells and DMEM medium for MDA-MB-231; all media were supplemented with $10 \%(\mathrm{v} / \mathrm{v})$ FBS and antibiotics $(100 \mu \mathrm{g} / \mathrm{mL}$ streptomycin and $100 \mathrm{UI} / \mathrm{mL}$ penicillin). All cultures were kept in a humidified incubator with $5 \% \mathrm{CO}_{2}$ at $37^{\circ} \mathrm{C}$.

2.4.2. Analysis of Cell Viability. Cell proliferation was measured by MTT assay based on the reduction of tetrazolium salt to formazan crystals by living cells [25]. Briefly, aliquots containing $1 \times 10^{4}(\mathrm{LNCaP})$ and $2 \times 10^{3}$ (MDA-MB-231) cells/well were seeded into 96-well plates. After $24 \mathrm{~h}$ of incubation at $37^{\circ} \mathrm{C}$ and $5 \% \mathrm{CO}_{2}$, freshly prepared solutions of free UA and SpHL-UA were added to the wells. The concentrations assayed were $1.2,2.5,5,10,20$, and $40 \mu \mathrm{M}$ of UA. Free UA was dissolved in DMSO prior to dilution. After $48 \mathrm{~h}$ of incubation at $37^{\circ} \mathrm{C}$ and $5 \% \mathrm{CO}_{2}$, the medium with treatment was removed and discarded. Subsequently, $100 \mu \mathrm{L}$ of culture medium containing $10 \%(\mathrm{v} / \mathrm{v})$ FBS and $0.5 \mathrm{mg}$ of tetrazolium $/ \mathrm{mL}$ was added to each well of the culture plate. After two hours of incubation, MTT crystals were solubilized in $100 \mu \mathrm{L}$ of a solution containing $10 \%(\mathrm{w} / \mathrm{v})$ SDS in $0.01 \mathrm{M}$ $\mathrm{HCl}$. Cell viability was estimated by measuring the rate of mitochondrial reduction of MTT determined by evaluating the absorbance of the converted dye at a wavelength of $595 \mathrm{~nm}$. Absorbance values of the wells in which the cells were maintained in medium alone were considered to be $100 \%$ of cell viability. The control groups included treatment with DMSO and blank liposomes. Data were expressed as percentage of cell viability compared to the control (mean \pm $\mathrm{SD}$ ). The $\mathrm{IC}_{50}$ values (i.e., UA concentration resulting in $50 \%$ of carcinoma cells viability) of free UA and SpHLUA were calculated using Graphpad Prism 5.0 (Graphpad Software Inc., San Diego, USA). At least three independent experiments were performed for each evaluated cancer cell line.

\section{Statistical Analysis}

Data were subjected to statistical analysis using the one-way analysis of variance (ANOVA), followed by the Bonferroni test, and $P$ values of less than 0.05 were regarded as significant (Graphpad Prism 5.0, Graphpad Software Inc., San Diego, USA). The results were expressed as mean values \pm standard deviation (S.D.).

\section{Results and Discussion}

4.1. HPLC Method Validation. The HPLC method showed adequate specificity. No interference of the liposome components could be identified, since no overlaps of peaks were detected after the injection of UA at the set wavelengths (data not shown). The linear response was obtained in the evaluated UA concentration range $(5-60 \mu \mathrm{g} / \mathrm{mL})$ with a correlation coefficient greater than 0.999 and a linear 
TABLE 1: Chemical and physicochemical properties of liposomes containing or not UA ${ }^{\mathrm{a}}$.

\begin{tabular}{|c|c|c|c|}
\hline Formulation & Mean diameter $(\mathrm{nm})$ & Zeta potential $(\mathrm{mV})$ & UA entrapment $(\mathrm{mg} / \mathrm{mL})$ \\
\hline SpHL $20 B^{b}$ & $105.8 \pm 3.3$ & $3.2 \pm 1.0$ & - \\
\hline SpHL 20UA1, & $191.1 \pm 6.4$ & $1.2 \pm 1.4$ & $0.77 \pm 0.01$ \\
\hline SpHL 20UA0.5 & $149.4 \pm 1.5$ & $1.7 \pm 2.6$ & $0.38 \pm 0.01$ \\
\hline SpHL $40 B^{\mathrm{e}}$ & $107.3 \pm 2.6$ & $2.9 \pm 0.4$ & - \\
\hline SpHL 40UA1 ${ }^{\mathrm{f}, \mathrm{h}}$ & $140.4 \pm 1.2$ & $4.1 \pm 1.3$ & $0.78 \pm 0.05$ \\
\hline SpHL $40 \mathrm{UA} 0.5^{\mathrm{g}, \mathrm{h}}$ & $122.8 \pm 3.1$ & $4.4 \pm 0.5$ & $0.35 \pm 0.04$ \\
\hline
\end{tabular}

${ }^{\mathrm{a}}$ Values are expressed as mean \pm S.D. $(n=3)$. ${ }^{\mathrm{b}}$ Lipid concentration is equal to $20 \mathrm{mM}$, without UA. ${ }^{\mathrm{c}}$ Lipid concentration is equal to $20 \mathrm{mM}$, and UA concentration is of $1 \mathrm{mg} / \mathrm{mL}$. ${ }^{\mathrm{d}}$ Lipid concentration is equal to $20 \mathrm{mM}$, and UA concentration is of $0.5 \mathrm{mg} / \mathrm{mL}$. ${ }^{\mathrm{e}}$ Lipid concentration is equal to $40 \mathrm{mM}$, without UA. ${ }^{\mathrm{f}}$ Lipid concentration is equal to $40 \mathrm{mM}$, and UA concentration is of $1 \mathrm{mg} / \mathrm{mL}$. ${ }^{\mathrm{g}}$ Lipid concentration is equal to $40 \mathrm{mM}$, and UA concentration is of $0.5 \mathrm{mg} / \mathrm{mL}$. ${ }^{\mathrm{h}}$ There is a significant difference among the mean diameter values of SpHL samples containing UA and the respective SpHL samples without UA at the same lipid concentration $(P<0.05)$.

TABLE 2: Distribution of vesicle diameter (\%) for SpHL 20UA1

\begin{tabular}{lcccc}
\hline Mean diameter $(\mathrm{nm})$ & \multicolumn{3}{c}{ Distribution of vesicle diameter $(\%)$} \\
& $\leq 100 \mathrm{~nm}$ & $\leq 300 \mathrm{~nm}$ & $300-500 \mathrm{~nm}$ & $\geq 500 \mathrm{~nm}$ \\
\hline $191.1 \pm 6.4$ & $72.4 \pm 14.7$ & $88.4 \pm 7.3$ & $7.1 \pm 4.7$ & $4.4 \pm 2.6$ \\
\hline
\end{tabular}

${ }^{\mathrm{a}}$ Each value represents the mean $\pm \operatorname{S.D}(n=3)$.

equation of $y=5001 x+2098$. The precision of the method was also confirmed. Whatever the UA concentration level, the overall results showed relative standard deviation values of lower than $5 \%$ in all experiments (data not shown). These results were in agreement with requirements for analytical assays [26].

4.2. SpHL-UA Characterization. The behavior of liposomes in storage conditions and biological mediums is determined by factors such as the size and surface charge of vesicles and the quantity of entrapped solute. Thus, it is of utmost importance to have as much information as possible regarding these parameters to ensure the efficacy and stabilization of the liposome formulation [27].

The chemical and physicochemical properties of SpHLUA, prepared with different lipid and drug concentrations, are summarized in Table 1.

Analyzing Table 1, it could be observed that the SpHL 20UA1 formulation mostly called attention due to its high concentration of UA embedded in the lowest level of lipid concentration $(20 \mathrm{mM})$. In addition, we can observe that the presence of UA leads to an increase in size of vesicles for all formulations. Moreover, by analyzing the distribution of vesicles of SpHL 20UA1, it could be observed that approximately $88 \%$ of the vesicles had to be smaller than $300 \mathrm{~nm}$ (Table 2). It is well known that liposomal preparations for anticancer treatment must present small-sized vesicles to comply with safety requirements and improve therapeutic efficacy [28]. Liposome size is extremely relevant to deliver anticancer agents to tumor tissue because these particles are known to accumulate in the tumor area due to the leaky vasculatureenhanced permeability and retention (EPR) effect. This effect occurs due to the anatomic differences between normal and cancerous tissue because capillaries in the tumor area possess increased permeability. This defective vascular architecture coupled with poor lymphatic drainage induces an enhanced permeability and retention. Therefore, liposomes in the range of 100 to $150 \mathrm{~nm}$ have been shown to preferentially accumulate in tumors due to this EPR effect. Large pores may exist in the tumor vessel wall that allow the penetration of liposomes up to the size of $400 \mathrm{~nm}$ in diameter [29-31]. Moreover, it is important to note that the size range is a compromise between loading efficiency of liposomes (increases with increasing size), liposome stability (decreases with increasing size above an optimal $80-200 \mathrm{~nm}$ range), and ability to extravasate (decreases with increasing size) [32]. Thus, the size of SpHL 20UA1 may contribute to their therapeutic success.

Therefore, SpHL 20UA1 was selected for subsequent studies. First, it was subjected to a stability study over a 60-day period. It is worth noting that SpHL 20UAl showed a good stability in terms of mean vesicle size, zeta potential, and UA entrapment after storage for 2 months at $4^{\circ} \mathrm{C}$ (Table 3 ).

A typical phenomenon of instability in the liposome formulation is the increase in particle size due to the aggregation or the fusion of unstable liposomes upon storage. An increase in particle size of liposomes generally results in a rapid uptake by MPS with a subsequent rapid clearance and a short halflife. Moreover, the fusion of vesicles leads to the leakage of the encapsulated drug. Thus, controlling and maintaining liposomes at small and uniform sizes are critical in developing a viable pharmaceutical product [33].

Zeta potential is an other important parameter to the stability of colloidal formulations. Generally, zeta potential values of $30 \mathrm{mV}$ (absolute value) and above characterize a stable formulation, since the aggregation of the particles is less likely to occur due to electrical repulsion forces [27]. However, in this work, as shown by zeta potential measurements, the liposome surface charges were near neutrality, but this seems not to have affected its stability (Table 3 ). The maintenance of vesicle diameter over time may well be due to the presence of PEG chains on the liposome surface, which 


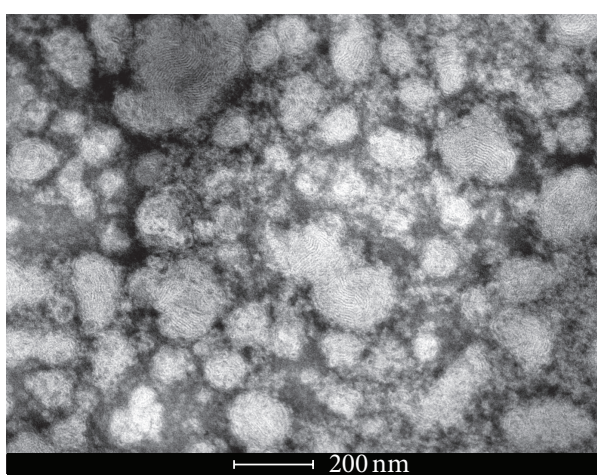

(a)

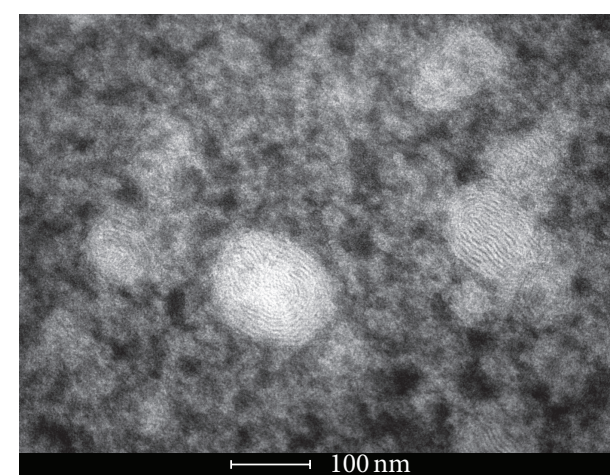

(b)

FIGURE 1: TEM photomicrographs obtained for SpHL 20UA1 in different fields ((a) and (b)).

TABLe 3: Physicochemical and chemical stability of SpHL 20UA1 ${ }^{\mathrm{a}}$.

\begin{tabular}{lccc}
\hline & Mean diameter $(\mathrm{nm})$ & Zeta potential $(\mathrm{mV})$ & UA entrapment $(\mathrm{mg} / \mathrm{mL})$ \\
\hline Time 0 & $191.1 \pm 6.4^{\mathrm{b}}$ & $1.2 \pm 1.4^{\mathrm{b}}$ & $0.77 \pm 0.01^{\mathrm{b}}$ \\
15 days & $210.3 \pm 15.2^{\mathrm{b}}$ & $-1.7 \pm 3.1^{\mathrm{b}}$ & $0.76 \pm 0.01^{\mathrm{b}}$ \\
30 days & $221.9 \pm 25.3^{\mathrm{b}}$ & $-1.6 \pm 3.5^{\mathrm{b}}$ & $0.76 \pm 0.01^{\mathrm{b}}$ \\
60 days & $212.7 \pm 15.3^{\mathrm{b}}$ & $-1.4 \pm 3.6^{\mathrm{b}}$ & $0.77 \pm 0.01^{\mathrm{b}}$ \\
\hline
\end{tabular}

${ }^{\mathrm{a}}$ Each value represents the mean \pm S.D $(n=3) .{ }^{\mathrm{b}}$ There is no significant difference among the means indicated at the same column $(P>0.05)$. All means were compared with time zero.

TABLE 4: $\mathrm{IC}_{50}$ values for cancer cell lines ${ }^{\mathrm{a}}$.

\begin{tabular}{lcc}
\hline Cell lines & $\mathrm{IC}_{50}(\mu \mathrm{M})$ & $\mathrm{IC}_{50}(\mu \mathrm{M})$ \\
& Free UA & SpHL 20UA1 \\
\hline MDA-MB-231 & $13.07 \pm 1.54^{\mathrm{b}}$ & $8.13 \pm 2.3$ \\
LNCaP & $2.49 \pm 0.53^{\mathrm{c}}$ & $2.68 \pm 1.06$ \\
\hline
\end{tabular}

${ }^{\mathrm{a}}$ Each value represents the mean \pm S.D $(n=3)$. ${ }^{\mathrm{b}}$ There is a significant difference among the means indicated at the same line $(P<0.05){ }^{c}$ There is no significant difference among the means indicated at the same line $(P>0.05)$.

could prevent vesicle aggregation, improving the stability of the formulations [18]. This fact can be explained by the steric repulsion provoked by the polymer chains of $\mathrm{PEG}_{2000}$-lipids [34].

Stability in terms of UA entrapment can be explained by the UA lipophilic character $(\log P=7.92)$ [35] which could enable a stronger interaction with phospholipids in a liposome bilayer, in turn preventing drug release during storage.

4.2.1. TEM Measurements. The images of SpHL 20UA1 obtained by TEM (Figure 1) allowed for the viewing of multilamellar vesicles of varying diameters, predominantly of vesicle sizes of less than $100 \mathrm{~nm}$, which were consistent with the results obtained from the particle sizes measured by PCS technique (shown in Table 2).

4.3. Cell Viability Assay. To evaluate the effects of SpHL 20UA1 on breast and prostate cancer cell lines viability, an MTT assay was employed. The $\mathrm{IC}_{50}$ values were calculated for each cell line and are summarized in Table 4. It is worth noting that effects on cell viability after control groups treatment (DMSO and blank liposomes) were negligible for both cancer cell lines.

In Table 4 , it can be observed that $\mathrm{IC}_{50}$ value obtained after SpHL 20UA1 treatment $(48 \mathrm{~h})$ was significantly lower than $\mathrm{IC}_{50}$ value obtained after UA free treatment $(P<0.05)$ for MDA-MB-231 cancer cell line. This result indicates that SpHL can improve UA delivery in this breast cancer cell line. There was not a significant difference among the $\mathrm{IC}_{50}$ values obtained after SpHL $20 \mathrm{UA1}$ and free UA treatments $(P>0.05)$ for LNCaP cancer cell line. These findings revealed that the incorporation of UA into SpHL did not provoke any impairment in the effect of UA on carcinoma cells viability. Thus, the use of SpHL 20UA1 may be a promising strategy to carry UA and allow for its intravenous administration.

\section{Conclusion}

In conclusion, the results of the present study demonstrated that SpHL-UA had a good stability in terms of mean vesicle size, zeta potential, and UA entrapment after storage for 2 months at $4^{\circ} \mathrm{C}$. Thus, the production of these liposomes proposed in this work led to liposomal dispersion with features suitable for future in vivo applications. This work also investigated the effects of SpHL-UA on breast and prostate cancer cell lines viability, which demonstrated that, after $48 \mathrm{~h}$, this treatment significantly inhibited cancer cell proliferation, as shown by MTT assay. Results from this study indicate that SpHL-UA can be an interesting delivery system for the pharmaceutical formulation of UA and may represent a good 
and useful chemotherapy agent for breast and prostate cancer treatment. Further evaluations, such as pharmacokinetics studies and antitumor activity in vivo, should be performed to confirm these expectations.

\section{Conflict of Interests}

The authors report no conflict of interests. The authors alone are responsible for the content and writing of the paper. Moreover, the paper has been proofread and revised by Todd Irwin Marshall, ELT Consultant and Executive Director, Pennsylvania English Consultoria e Traduções LTDA.

\section{Acknowledgments}

The authors wish to thank CAPES (Rede NanobiotecBrasil), FAPEMIG, and CNPq for their financial support. The authors would also like to acknowledge the Centro de Microscopia da Universidade Federal de Minas Gerais (http://www.microscopia.ufmg.br) for providing the equipment and technical support for experiments involving electron microscopy. The authors would also wish to thank Professor Alfredo Miranda de Goes for providing cancer cell line MDA-MB-231.

\section{References}

[1] R. Gautam and S. M. Jachak, "Recent developments in antiinflammatory natural products," Medicinal Research Reviews, vol. 29, no. 5, pp. 767-820, 2009.

[2] S. H. Kim, J. H. Hong, and Y. C. Lee, "Ursolic acid, a potential PPARg agonist, suppresses ovalbumin-induced airway inflammation and Penh by down-regulating IL-5, IL-13, and IL-17 in a mouse model of allergic asthma," European Journal of Pharmacology, vol. 701, no. 1-3, pp. 131-143, 2013.

[3] L. Jie, "Pharmacology of oleanolic acid and ursolic acid," Journal of Ethnopharmacology, vol. 49, pp. 57-68, 1995.

[4] L. O. Somova, A. Nadar, P. Rammanan, and F. O. Shode, "Cardiovascular, antihyperlipidemic and antioxidant effects of oleanolic and ursolic acids in experimental hypertension," Phytomedicine, vol. 10, no. 2-3, pp. 115-121, 2003.

[5] I. Bonaccorsi, F. Altieri, I. Sciamanna et al., "Endogenous reverse transcriptase as a mediator of ursolic acid's antiproliferative and differentiating effects in human cancer cell lines," Cancer Letters, vol. 263, no. 1, pp. 130-139, 2008.

[6] K. A. Manu and G. Kuttan, "Ursolic acid induces apoptosis by activating p53 and caspase-3 gene expressions and suppressing NF- $\kappa$ B mediated activation of bcl-2 in B16F-10 melanoma cells," International Immunopharmacology, vol. 8, no. 7, pp. 974-981, 2008.

[7] C. P. R. Xavier, C. F. Lima, A. Preto, R. Seruca, M. FernandesFerreira, and C. Pereira-Wilson, "Luteolin, quercetin and ursolic acid are potent inhibitors of proliferation and inducers of apoptosis in both KRAS and BRAF mutated human colorectal cancer cells," Cancer Letters, vol. 281, no. 2, pp. 162-170, 2009.

[8] E. Kassi, T. G. Sourlingas, M. Spiliotaki et al., "Ursolic acid triggers apoptosis and Bcl-2 downregulation in MCF-7 breast cancer cells," Cancer Investigation, vol. 27, no. 7, pp. 723-733, 2009.
[9] S.-L. Yan, C.-Y. Huang, S.-T. Wu, and M.-C. Yin, "Oleanolic acid and ursolic acid induce apoptosis in four human liver cancer cell lines," Toxicology in Vitro, vol. 24, no. 3, pp. 842-848, 2010.

[10] C.-Y. Huang, C.-Y. Lin, C.-W. Tsai, and M.-C. Yin, "Inhibition of cell proliferation, invasion and migration by ursolic acid in human lung cancer cell lines," Toxicology in Vitro, vol. 25, no. 7, pp. 1274-1280, 2011.

[11] K. H. Kim, H. S. Seo, H. S. Choi, I. H. Choi, Y. C. Shin, and S.-G. Ko, "Induction of apoptotic cell death by ursolic acid through mitochondrial death pathway and extrinsic death receptor pathway in MDA-MB-231 cells," Archives of Pharmacal Research, vol. 34, no. 8, pp. 1363-1372, 2011.

[12] H. Zhang, X. Li, and J. Ding, "Delivery of ursolic acid (UA) in polymeric nanoparticles effectively promotes the apoptosis of gastric cancer cells through enhanced inhibition of cyclooxygenase 2 (COX-2)," International Journal of Pharmaceutics, vol. 441, no. 1-2, pp. 261-268, 2013.

[13] J. Liu, "Oleanolic acid and ursolic acid: research perspectives," Journal of Ethnopharmacology, vol. 100, no. 1-2, pp. 92-94, 2005.

[14] Y. Zhong, J. Wang, Y. Wang et al., "Preparation and evaluation of liposome-encapsulated codrug LMX," International Journal of Pharmaceutics, vol. 438, pp. 240-248, 2012.

[15] S. Vemuri and C. T. Rhodes, "Preparation and characterization of liposomes as therapeutic delivery systems: a review," Pharmaceutica Acta Helvetiae, vol. 70, no. 2, pp. 95-111, 1995.

[16] J. Huwyler, J. Drewe, and S. Krähenbühl, “Tumor targeting using liposomal antineoplastic drugs," International Journal of Nanomedicine, vol. 3, no. 1, pp. 21-29, 2008.

[17] R. Cavalli, O. Caputo, and M. R. Gasco, "Preparation and characterization of solid lipid nanospheres containing paclitaxel," European Journal of Pharmaceutical Sciences, vol. 10, no. 4, pp. 305-309, 2000.

[18] M. L. Immordino, F. Dosio, and L. Cattel, "Stealth liposomes: review of the basic science, rationale, and clinical applications, existing and potential," International Journal of Nanomedicine, vol. 1, no. 3, pp. 297-315, 2006.

[19] V. Torchilin, "Multifunctional and stimuli-sensitive pharmaceutical nanocarriers," European Journal of Pharmaceutics and Biopharmaceutics, vol. 71, no. 3, pp. 431-444, 2009.

[20] M. C. De Oliveira, V. Rosilio, P. Lesieur et al., "pH-sensitive liposomes as a carrier for oligonucleotides: a physico-chemical study of the interaction between DOPE and a 15-mer oligonucleotide in excess water," Biophysical Chemistry, vol. 87, no. 2-3, pp. 127-137, 2000.

[21] S. Simões, J. Nuno Moreira, C. Fonseca, N. Düzgüneş, and M. C. Pedroso De Lima, "On the formulation of $\mathrm{pH}$-sensitive liposomes with long circulation times," Advanced Drug Delivery Reviews, vol. 56, no. 7, pp. 947-965, 2004.

[22] A. D. Carvalho Jr., F. P. Vieira, V. J. De Melo et al., "Preparation and cytotoxicity of cisplatin-containing liposomes," Brazilian Journal of Medical and Biological Research, vol. 40, no. 8, pp. 1149-1157, 2007.

[23] P. M. Gullino, F. H. Grantham, S. H. Smith, and A. C. Haggerty, "Modifications of the acid-base status of the internal milieu of tumors," Journal of the National Cancer Institute, vol. 34, no. 6, pp. 857-869, 1965.

[24] A. D. Bangham, M. M. Standish, and J. C. Watkins, "Diffusion of univalent ions across the lamellae of swollen phospholipids," Journal of Molecular Biology, vol. 13, no. 1, pp. 238-252, 1965.

[25] T. Mosmann, "Rapid colorimetric assay for cellular growth and survival: application to proliferation and cytotoxicity assays," 
Journal of Immunological Methods, vol. 65, no. 1-2, pp. 55-63, 1983.

[26] Agência Nacional De Vigilância Sanitária, "Guia para validação de métodos analíticos," Resolução no. 899, de 20 de maio de 2003, http://www.anvisa.gov.br/legis/resol/2003/re/ 899_03re.htm.

[27] R. R. C. New, Liposomes: A Pratical Approach, Oxford University Press, New York, NY, USA, 1990.

[28] V. P. Torchilin, "Targeted pharmaceutical nanocarriers for cancer therapy and imaging," The AAPS Journal, vol. 9, no. 2, pp. E128-E147, 2007.

[29] F. Yuan, M. Dellian, D. Fukumura et al., "Vascular permeability in a human tumor xenograft: molecular size dependence and cutoff size," Cancer Research, vol. 55, no. 17, pp. 3752-3756, 1995.

[30] X. Wang, Y. Wang, Z. Chen et al., "Advances of cancer therapy by nanotechnology," Cancer Research and Treatment, vol. 41, pp. 1-11, 2009.

[31] A. Puri, K. Loomis, B. Smith et al., "Lipid-based nanoparticles as pharmaceutical drug carriers: from concepts to clinic," Critical Reviews in Therapeutic Drug Carrier Systems, vol. 26, no. 6, pp. 523-580, 2009.

[32] D. D. Lasic, "Novel applications of liposomes," Trends in Biotechnology, vol. 16, no. 7, pp. 307-321, 1998.

[33] T. Yang, F.-D. Cui, M.-K. Choi et al., "Enhanced solubility and stability of PEGylated liposomal paclitaxel: in vitro and in vivo evaluation," International Journal of Pharmaceutics, vol. 338, no. 1-2, pp. 317-326, 2007.

[34] M. Johnsson and K. Edwards, "Liposomes, disks and spherical micelles: aggregate structure in mixtures of gel phase phosphatidylcholines and poly(ethyleneglycol)-phospholipids," Biophysical Journal, vol. 85, no. 6, pp. 3839-3847, 2003.

[35] A. A. Ramos, C. F. Lima, M. L. Pereira, M. Fernandes-Ferreira, and C. Pereira-Wilson, "Antigenotoxic effects of quercetin, rutin and ursolic acid on HepG2 cells: evaluation by the comet assay," Toxicology Letters, vol. 177, no. 1, pp. 66-73, 2008. 

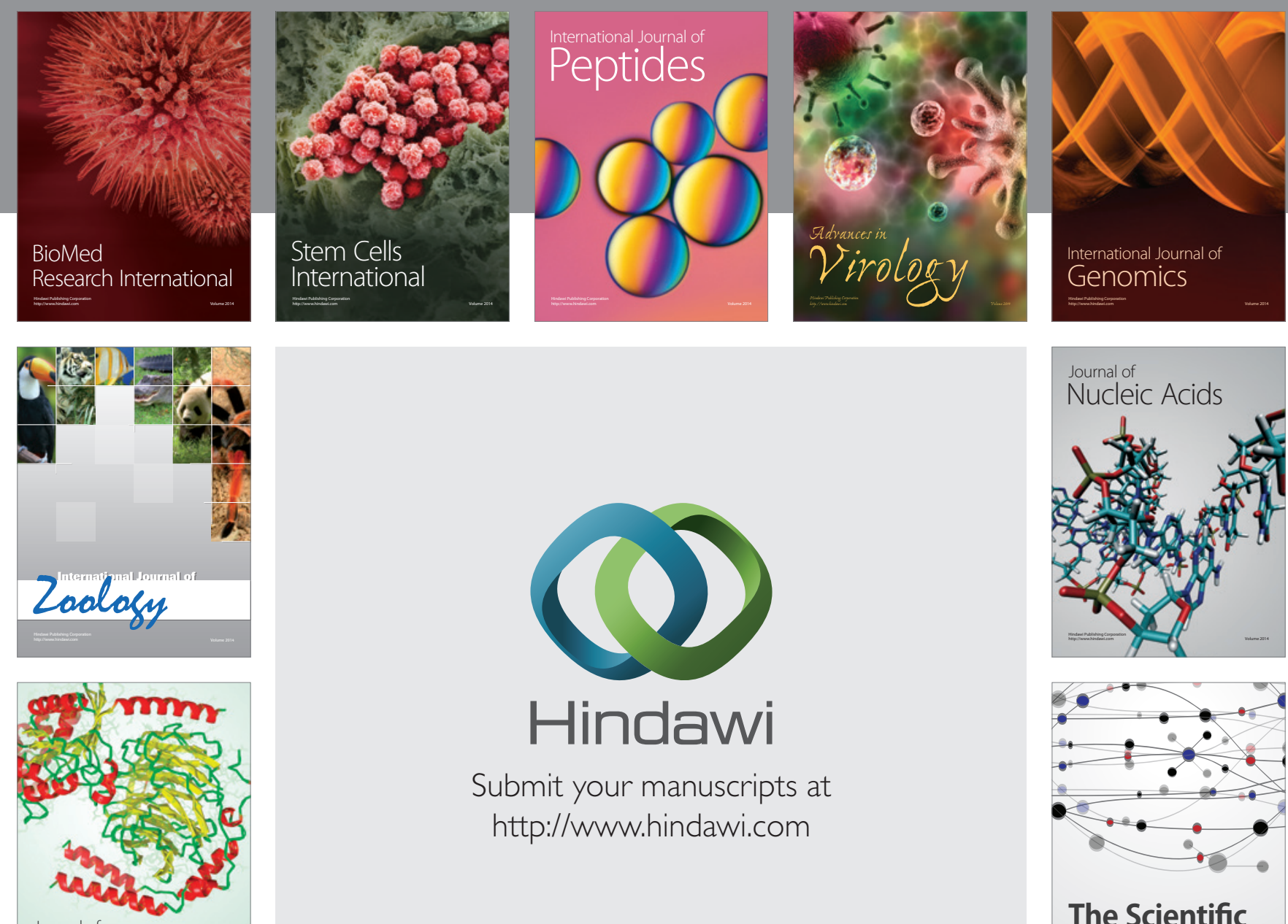

Submit your manuscripts at

http://www.hindawi.com

Journal of
Signal Transduction
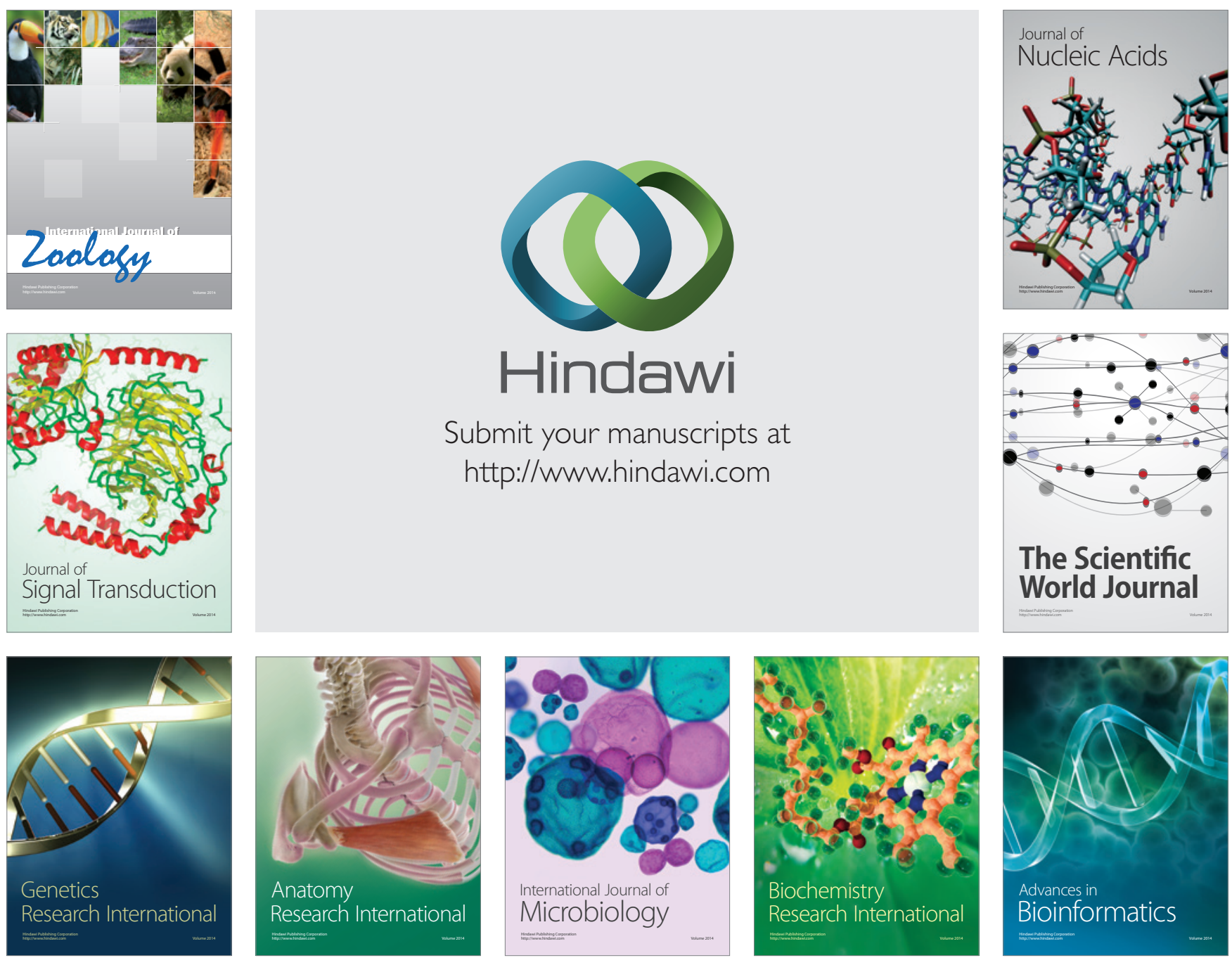

The Scientific World Journal
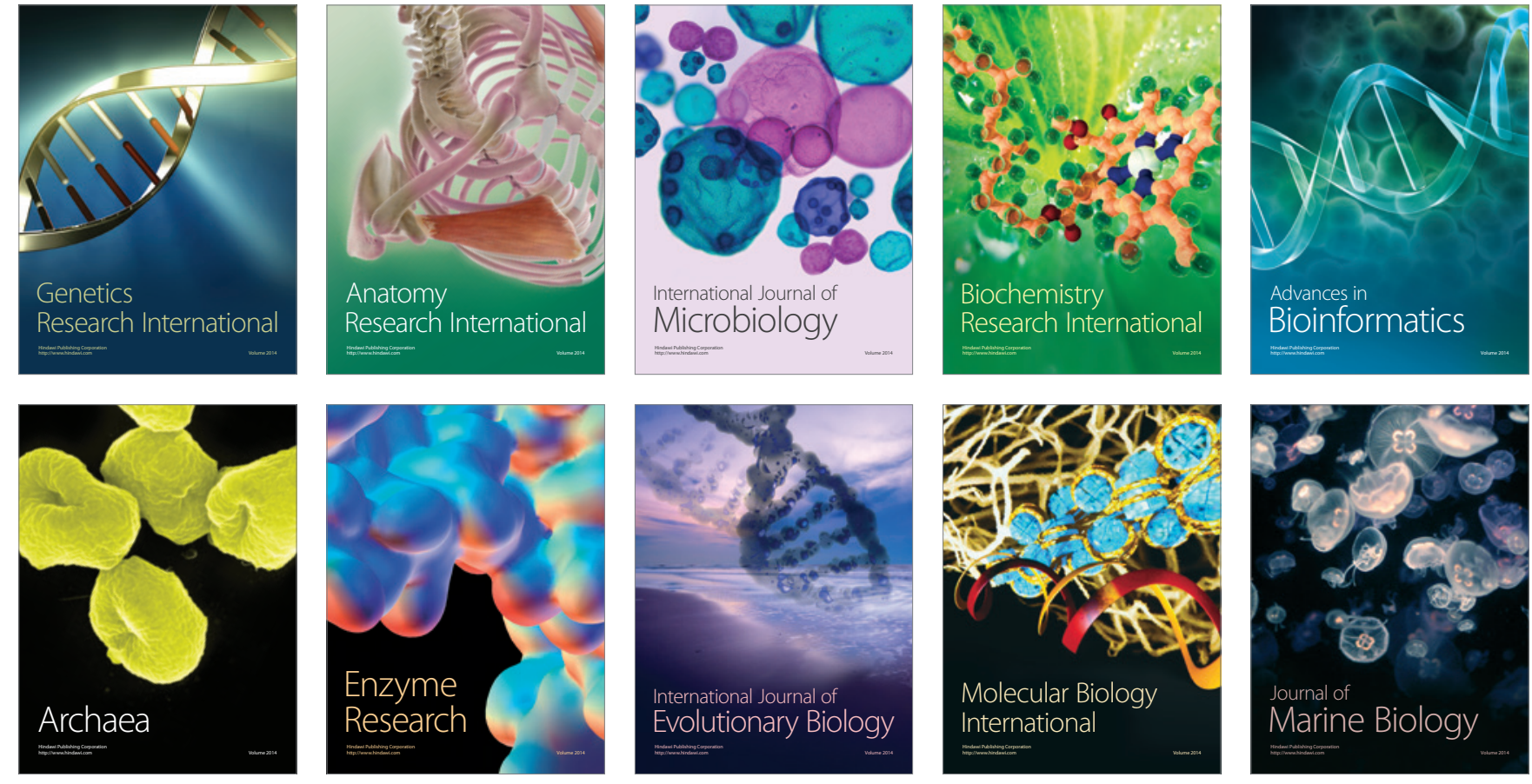